\title{
ALTERNATIVAS RECONSTRUCTIVAS POST MAXILECTOMÍA POR ENFERMEDAD NEOPLÁSICA*
}

\author{
Drs. Ricardo Yáñez M. ${ }^{1}$, Francisco Javier Loyola B. ${ }^{1,2}$, Diego Alcocer C. ${ }^{1}$, \\ Jorge Cornejo F. ${ }^{1}$, Mariana Valenzuela G. ${ }^{1}$, Raúl Martínez R. ${ }^{1,2}$ \\ 1 Cirugía de Cabeza y Cuello, Servicio de Cirugía, Hospital Dr. Sótero del Río. \\ 2 Pontificia Universidad Católica de Chile. \\ Chile.
}

\begin{abstract}
\section{Reconstructive alternatives post maxillectomy for oncologic disease}

Background: The reconstruction of midfacial defects is a major challenge. The maxillary bones are the more important of the facial skeleton, provide support between skull base and maxillary arches, separate cavities and are involved in swallowing, phonation, mastication, vision and appearance. The maxillectomy involves varying degrees of functional impairment. The maxillary reconstruction ranges from the use of obturator prosthesis, local flaps to free flaps. Aim: To present the different reconstructive alternatives used after a maxillectomy for oncologic disease in our hospital. Patients and Methods: Retrospective analysis of all patients that was submitted to a maxillectomy for oncologic disease between 2008 and 2011 in our center. Results: The series consisted of 12 patients, 8 women and median age 57 years (range, 25-84). We realized Type IIA maxillectomy in five patients, IIB to two patients, IIIA to four patients and IIIB to one patient. We achieved R0 in all cases. The reconstruction was realized with obturator prosthesis in four patients, three patients with pedicled flaps and five patients were reconstructed with free flaps. All patients obtain an adequate functional status. One patient has partial flap loss. Discussion: Is recommended adjust the surgical choice to the patient prognosis. The obturator prosthesis is a good choice for selected group. The temporal muscle flap presents adequate functional results and is recommended in advanced disease and poor prognosis. The microsurgical reconstruction is the best choice with better functional and aesthetics outcomes in type II, III and IV maxillectomy.

Key words: Maxillectomy, reconstructive surgery, free flap, microsurgery.

\section{Resumen}

Introducción: La reconstrucción de defectos mediofaciales representa un gran desafío. Los maxilares son los huesos más importantes del esqueleto facial, proveen soporte entre base del cráneo y arcos maxilares, separan cavidades, participan en la deglución, fonación, masticación, visión y apariencia. La maxilectomía conlleva diferentes grados de alteración funcional. La reconstrucción maxilar va desde el uso de prótesis obturadoras, colgajos locales hasta colgajos libres. Objetivo: Presentar las alternativas reconstructivas post

*Recibido el 30 de abril de 2013 y aceptado para publicación el 15 de julio de 2013.

Los autores no refieren conflictos de interés.

Correspondencia: Dr. Ricardo Yáñez M.

Av. Concha y Toro $3459,5^{\circ}$ piso, Santiago, Chile. Fax: $+56-2-23536601$. yanezmartinez@gmail.com 
maxilectomía por enfermedad neoplásica utilizadas en nuestro centro. Pacientes y Métodos: Análisis retrospectivo de todos los pacientes sometidos a maxilectomía por causa oncológica entre los años 2008 y 2011. Resultados: La serie estuvo compuesta por 12 pacientes, 8 mujeres, mediana de edad 57 años (rango 25-84). Se realizó maxilectomía Tipo IIA a cinco pacientes, Tipo IIB a dos pacientes, Tipo IIIA a cuatro pacientes y IIIB a un paciente. Se logró R0 en todos los casos. La reconstrucción se realizó con prótesis obturadora en cuatro pacientes, tres pacientes con colgajos pediculados y cinco pacientes con colgajos microquirúrgicos. En todos los pacientes se logró un resultado funcional adecuado. En un paciente hubo pérdida parcial del colgajo. Discusión: Es recomendable ajustar la alternativa reconstructiva según el pronóstico del paciente. Prótesis obturadoras son una opción para pacientes seleccionados. El colgajo de músculo temporal presenta un buen resultado funcional, recomendado en pacientes con enfermedad avanzada y mal pronóstico. La reconstrucción microquirúrgica es la alternativa de elección con los mejores resultados funcionales y estéticos en pacientes con maxilectomías Tipo II, III y IV.

Palabras clave: Maxilectomía, cirugía reconstructiva, colgajo libre, microcirugía.

\section{Introducción}

La reconstrucción mediofacial es una de las más complejas y uno de los mayores desafíos en cirugía reconstructiva de cabeza y cuello. Ambos maxilares son las estructuras óseas más importantes de la región facial y cumplen distintas funciones tales como separación de cavidades oral y sinonasal, soportan el contenido orbitario, permiten el habla y contribuyen en la apariencia estética ${ }^{1,2}$.

Los defectos maxilares pueden ser congénitos o secundarios a traumatismos, pero principalmente se deben a cirugía resectiva oncológica por tumores que comprometen el maxilar y tejidos adyacentes en la región mediofacial, el paladar o la órbita. En este tipo de pacientes es necesario resecar el tumor y realizar la cobertura de forma adecuada de modo que pueda realizarse la radioterapia y el tratamiento postoperatorio ${ }^{3}$.

Los principios reconstructivos en defectos mediofaciales incluyen cierre y obliteración del defecto post maxilectomía, dar sostén al globo ocular si es preservado, de lo contrario realizar llene de la cavidad orbitaria en caso de exanteración ocular, mantener una barrera entre senos nasales y fosa craneal anterior, restaurar apariencia facial y reconstruir el paladar ${ }^{4}$.

La reconstrucción maxilar va desde el uso de prótesis obturadoras, colgajos locales, colgajos pediculados hasta colgajos microquirúrgicos dependiendo del tipo de defecto y del pronóstico del paciente ${ }^{4,5}$.

El objetivo del presente estudio es presentar las distintas alternativas reconstructivas post maxilectomías por causa oncológica utilizadas en nuestro centro.

\section{Pacientes y Método}

\section{Pacientes}

Estudio retrospectivo, descriptivo. Se realizó un análisis de todos los pacientes con tumores maxilares que fueron sometidos a maxilectomía por el equipo de Cirugía de Cabeza y Cuello en el Hospital Dr. Sótero del Río entre los años 2008 y 2011. Se registró datos demográficos de cada paciente, características del tumor primario, etapificación según TNM, y Tipo de maxilectomía realizada ${ }^{6}$. Se logró el seguimiento de todos los pacientes.

Las complicaciones postoperatorias tempranas fueron definidas como aquellas que se presentaron dentro de los primeros 90 días desde la cirugía y tardías aquellas que se presentaron posterior a este período ${ }^{7}$. Se definió como complicación menor pérdida parcial del colgajo, infección de partes blandas, hematoma y sangrado; se definió como complicación mayor patología sistémica, pérdida total del colgajo y muerte del paciente.

Los pacientes que fueron sometidos a maxilectomía estaban en buenas condiciones generales y no presentaban enfermedad diseminada. Fueron evaluados con Tomografía Axial Computada de Cabeza y Cuello.

\section{Maxilectomía}

Se utilizó la clasificación anatómica para maxilectomías de Cordeiroํ. Se definió Maxilectomía Tipo I o parcial aquella que compromete una o dos paredes del maxilar, más frecuentemente las paredes anterior y medial. Tipo II o maxilectomía subtotal la resección de las cinco paredes inferiores del maxilar sin comprometer el piso orbitario; Tipo IIA aquellas que incluyen menos del $50 \%$ del paladar duro y Tipo IIB aquellas que incluyen más del 50\% del paladar duro. Tipo III son aquellas maxilectomías totales que incluyen la resección de las seis paredes del maxilar; se subdividen en Tipo IIIA (no incluye contenido orbitario) y IIIB (incluye contenido orbitario). Tipo IV compromete las cinco paredes superiores del maxilar, incluyendo la órbita y su contenido, dejando expuesto cerebro y duramadre. 


\section{Resultado funcional y estético}

Para evaluar el resultado funcional se objetivó fonación, dieta tolerada, competencia oral y presencia de fístula oronasal. Fue realizado por encuesta durante el control clínico.

La evaluación estética se realizó mediante la evaluación de fotografías del paciente por dos observadores (cirujanos) externos.

\section{Resultados}

La serie estuvo compuesta por 12 pacientes, ocho mujeres. La mediana de edad fue de 57 años (rango, 25-84 años). En 8 pacientes se obtuvo como antecedente el tabaquismo y en 5 pacientes un consumo excesivo de alcohol.

En 7 pacientes el motivo de consulta fue derivación desde odontología seguido por dolor y/o aumento de volumen en 5 pacientes. Las características generales de los pacientes y la clasificación según TNM se encuentra detallada en la Tabla 1.

Se realizó una maxilectomía Tipo II a 7 pacientes, Tipo IIA a 5 pacientes, Tipo IIB a 2 pacientes, una maxilectomía Tipo IIIA a 4 pacientes y IIIB a un paciente (Figuras 1-4). Los diagnósticos histológicos se presentan en la Tabla 2. Se logró márgenes microscópicos negativos (R0) en todos los enfermos.

Según el tipo de maxilectomía y las características propias de cada uno de los pacientes se realizó distintos tipos de reconstrucción: de los 12 pacientes, a 4 se les realizó maxilectomía y reconstrucción con prótesis obturadora, 3 pacientes fueron reconstruidos con colgajos pediculados, 5 pacientes con colgajos microquirúrgicos, el detalle se observa en la Tabla 3.

En el período de 90 días comprendidos desde la realización de la maxilectomía se registró mortalidad en un paciente por neumonía aspirativa. Se registró complicaciones mayores en 2 pacientes, ambos con neumonías aspirativas y uno con fallecimiento por esta causa. Se registró complicaciones menores en un paciente con pérdida parcial del colgajo (latissimus dorsi) por trombosis venosa.

Tabla 1. Características de los pacientes con tumores maxilares sometidos a maxilectomía

\begin{tabular}{|lll|}
\hline Características de los pacientes & $\mathbf{n}=\mathbf{1 2}$ & \\
\hline Edad (mediana y rango) & $\begin{array}{l}57 \text { años } \\
\text { (rango 25-84) }\end{array}$ & \\
Género femenino:masculino & $8: 4$ & $75 \%$ \\
Tabaquismo & 8 & $41,6 \%$ \\
Alcoholismo & 5 & \\
Motivo de consulta & & $41,6 \%$ \\
Aumento de volumen/dolor & 5 & $58,4 \%$ \\
Derivado odontología & 7 & \\
TNM & & \\
$\quad$ Estadío I & & \\
$\quad$ Estadío II & & \\
$\quad$ Estadío III & & \\
$\quad$ Estadío IV & 100 & \\
\hline
\end{tabular}
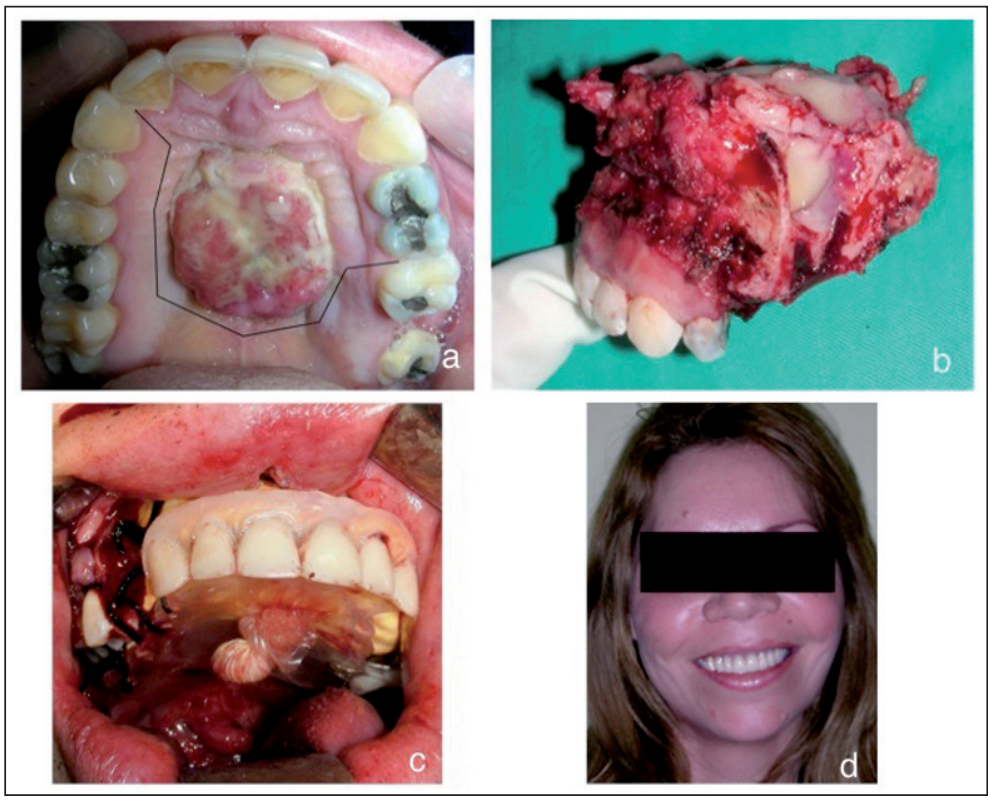

Figura 1. Sarcoma de paladar, se realizó una maxilectomía Tipo IIA y reconstrucción con una prótesis obturadora. a: Marcación preoperatoria del tumor y resección a realizar. b: Pieza operatoria, tumor resecado con margen libre de tumor. c: Inserción de prótesis obturadora en el defecto. d: Control postoperatorio a un año. 

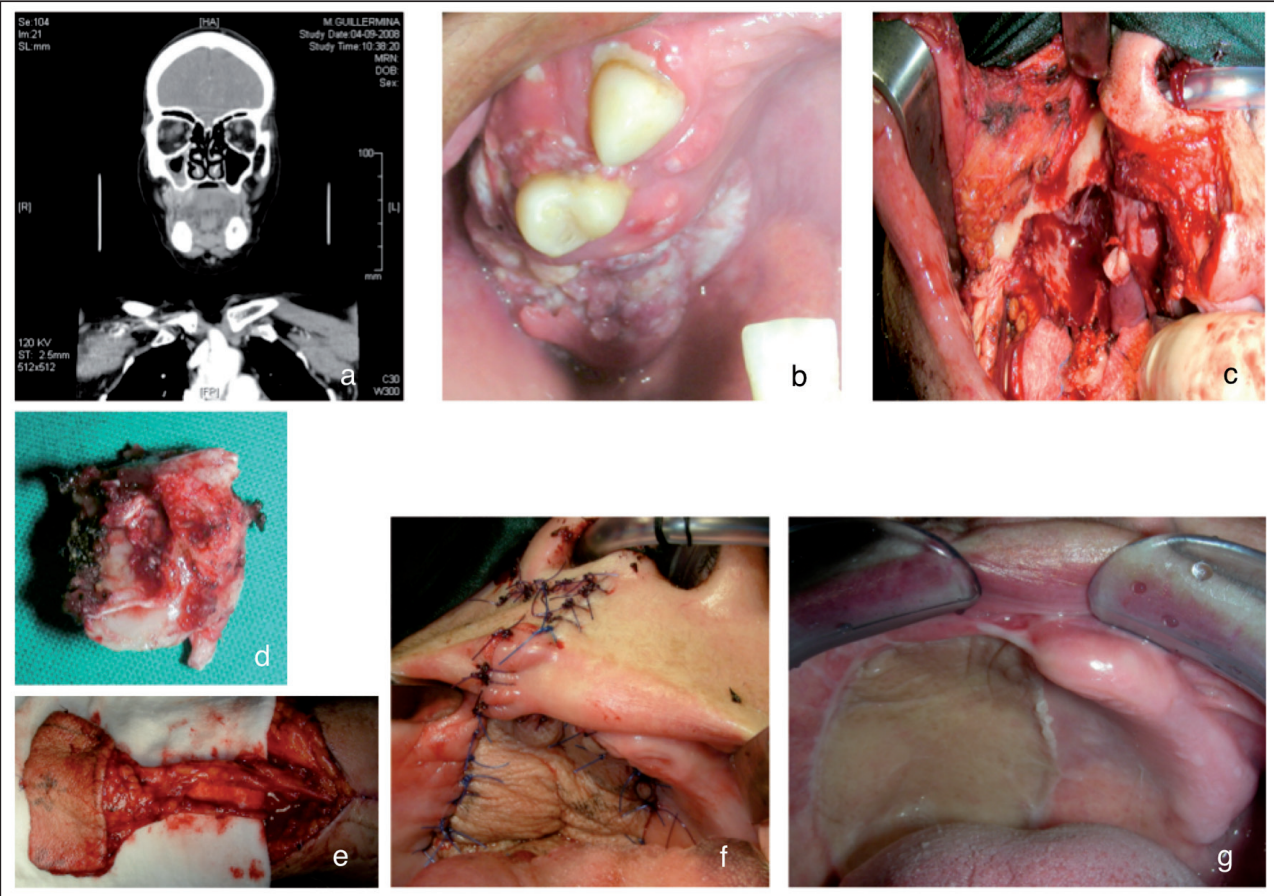

Figura 2. Tumor de seno maxilar, se realizó una maxilectomía Tipo IIB y reconstrucción con colgajo radial. a: Tomografía preoperatoria donde se observa compromiso tumoral del seno maxilar izquierdo, que correspondió en la biopsia a un carcinoma escamoso. b: Lesión de maxilar por la cual consulta el paciente. c: Defecto post maxilectomía que incluye más del 50\% del paladar. d: Pieza operatoria, tumor maxilar resecado con bordes negativos. e: Colgajo radial. f: Colgajo radial cubriendo defecto maxilar. g: Control postoperatorio a los 6 meses.

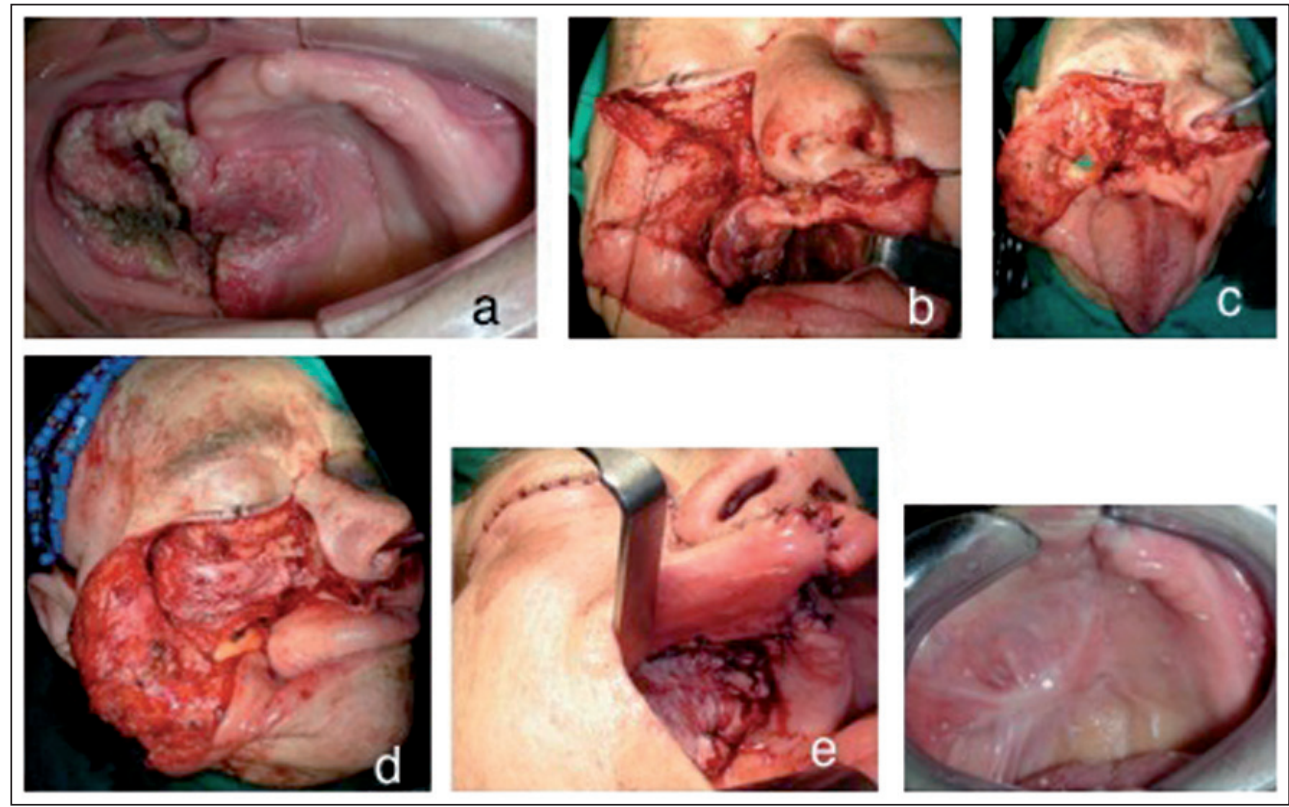

Figura 3. Tumor de seno maxilar, se realizó una maxilectomía Tipo IIIA y reconstrucción con colgajo de músculo temporal. a: Lesión de maxilar, preoperatorio. b: Abordaje de Weber-Ferguson. c: Defecto Tipo IIIA. d: Cobertura con colgajo de músculo temporal. e: Postoperatorio inmediato. f: Control postoperatorio a los 6 meses. 

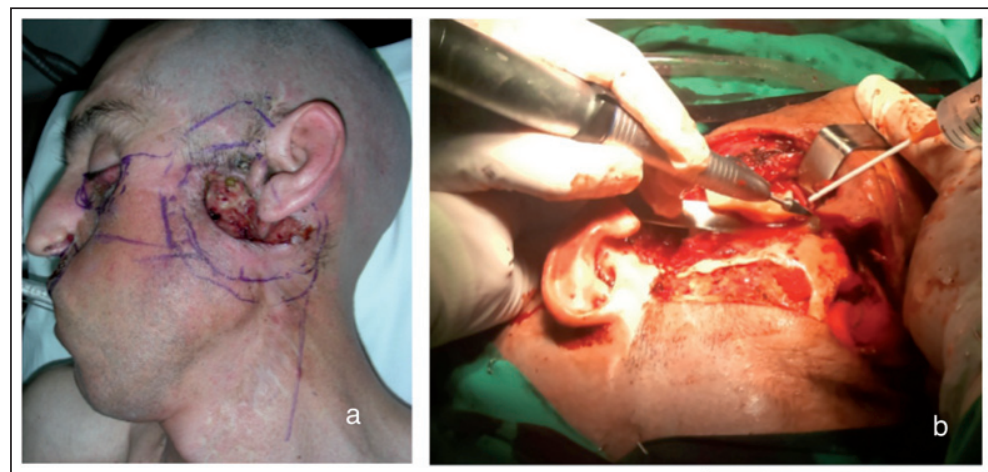

Figura 4. Cáncer basocelular de

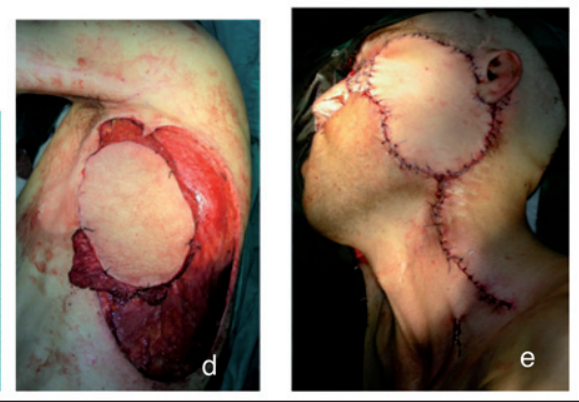
mejilla recidivado, con compromiso maxilar. Se realizó una maxilectomía Tipo IIIB y reconstrucción con colgajo libre de latissimus dorsi. a: Visión preoperatoria de tumor que compromete mejilla y maxilar. b: Intraoperatorio, resección de pared lateral de maxilar y exanteración orbitaria. c: Pieza operatoria. d: Disección de colgajo latissimus dorsi. e: Postoperatorio inmediato una vez realizado el inset del colgajo.

Tabla 2. Distribución porcentual según diagnóstico patológico

\begin{tabular}{|lcc|}
\hline Diagnóstico patológico & n & \% \\
\hline Carcinoma escamoso & 6 & 50 \\
Melanoma & 2 & 16,7 \\
\hline Sarcoma & 2 & 16,7 \\
\hline Carcinoma adenoideo quístico & 1 & 8,3 \\
\hline Cáncer basocelular & 1 & 8,3 \\
\hline
\end{tabular}

La realimentación oral se inició al tercer día postoperatorio en los pacientes sin compromiso de la cavidad oral y al séptimo día en aquellos con compromiso de ésta. La estadía hospitalaria fue de 15 días (rango 7-28 días).

Hubo recidiva de la enfermedad en una paciente con melanoma de paladar, con enfermedad recurrente local y a distancia.

Desde el punto de vista funcional y estético la mayoría de los pacientes tuvo una fonación y dieta normal, el resultado estético en la mayoría fue bueno $\mathrm{y}$ todos los pacientes mantuvieron competencia oral, ningún paciente presentó fístula oronasal (Tabla 4).

Tabla 3. Distribución según tipo de defecto y reconstrucción realizada

\begin{tabular}{|cccccc|}
\hline $\begin{array}{c}\text { Tipo de } \\
\text { maxilectomía }\end{array}$ & Total & Colgajo radial* & $\begin{array}{c}\text { Colgajo } \\
\text { latissimus dorsi* }\end{array}$ & $\begin{array}{c}\text { Colgajo músculo } \\
\text { temporal** }\end{array}$ & $\begin{array}{c}\text { Prótesis } \\
\text { obturadora }\end{array}$ \\
\hline I & 0 & - & - & - & 0 \\
IIA & 5 & 1 & - & - & 4 \\
\hline IIB & 2 & 2 & - & - & - \\
IIIA & 4 & - & 1 & 3 & - \\
IIIB & 1 & - & 1 & - & - \\
IV & - & - & - & - & - \\
Total & 12 & 3 & 2 & 3 & 4 \\
\hline
\end{tabular}

*Colgajo libre. **Colgajo pediculado. 
Tabla 4. Resultado funcional y estético

\begin{tabular}{|c|c|c|c|c|c|}
\hline Tipo de maxilectomía & $\begin{array}{c}\text { Tipo IIA } \\
(n=5)\end{array}$ & $\begin{array}{c}\text { Tipo IIB } \\
(n=2)\end{array}$ & $\begin{array}{c}\text { Tipo IIIA } \\
(\mathrm{n}=4)\end{array}$ & $\begin{array}{c}\text { Tipo IIIB } \\
(\mathrm{n}=1)\end{array}$ & $\begin{array}{c}\text { Total } \\
(n=12)\end{array}$ \\
\hline $\begin{array}{l}\text { Fonación } \\
\text { Normal } \\
\text { Inteligible } \\
\text { Ininteligible }\end{array}$ & $\begin{array}{l}3 \\
2\end{array}$ & $\begin{array}{l}1 \\
1\end{array}$ & $\begin{array}{l}1 \\
3\end{array}$ & 1 & $\begin{array}{ll}6 & (50 \%) \\
6 & (50 \%)\end{array}$ \\
\hline $\begin{array}{l}\text { Dieta } \\
\text { Normal } \\
\text { Blando } \\
\text { Líquida }\end{array}$ & $\begin{array}{l}3 \\
2\end{array}$ & $\begin{array}{l}1 \\
1\end{array}$ & $\begin{array}{l}1 \\
2 \\
1\end{array}$ & 1 & $\begin{array}{ll}6 & (50 \%) \\
5 & (41,6 \%) \\
1 & (8,3 \%)\end{array}$ \\
\hline $\begin{array}{l}\text { Resultado estético } \\
\text { Excelente } \\
\text { Bueno } \\
\text { Regular } \\
\text { Malo }\end{array}$ & $\begin{array}{l}1 \\
4\end{array}$ & $\begin{array}{l}1 \\
1\end{array}$ & $\begin{array}{l}2 \\
1 \\
1\end{array}$ & 1 & $\begin{array}{rr}2 & (16,6 \%) \\
8 & (66,6 \%) \\
1 & (8,3 \%) \\
1 & (8,3 \%)\end{array}$ \\
\hline $\begin{array}{l}\text { Competencia oral } \\
\text { Sí } \\
\text { No }\end{array}$ & 5 & 2 & 4 & 1 & $12(100 \%)$ \\
\hline $\begin{array}{l}\text { Fístula oro nasal } \\
\text { Sí } \\
\text { No }\end{array}$ & 5 & 2 & 4 & 1 & $12(100 \%)$ \\
\hline
\end{tabular}

\section{Discusión}

Uno de los tumores más agresivos de cabeza y cuello que comprometen la región mediofacial es el carcinoma escamoso. Una amplia variedad de otros tumores que va desde cáncer basocelular hasta carcinoma adenoideo quístico infiltrativo pueden resultar altamente agresivos, asimismo el melanoma maligno ha comenzado a ser cada vez más prevalente y una importante causa de tumores que comprometen el maxilar. El tratamiento quirúrgico de este tipo de tumores es en general la resección amplia con márgenes libres de enfermedad ${ }^{3,8}$.

Las resecciones maxilares, ya sean subtotales o totales, por su extensión y ubicación son de una alta complejidad tanto para realizarlas como para reconstruir el defecto, ya que comprometen desde el oído y cavidades nasales hasta la cavidad oral, por lo cual restaurar función y forma es un verdadero desafío, más aún sabiendo que la mayoría de estos pacientes requerirá radioterapia en el postoperatorio ${ }^{9}$.

Existen distintos sistemas para clasificar las maxilectomías, una es la clasificación de Selibeau en 1906, quien describió formas clínicas de cáncer maxilar y dio una descripción de la ruta de diseminación tumoral, además dividió la región facial en supra, meso e infraestructura. Posteriormente, en 1925, Cornet dividió el maxilar en tres partes: supraestructura (el complejo etmoido-máxilo-orbito- malar), una mesoestructura nasosinusal y la infraestructura: el paladar. Georg Öhngren en 1933, dividió la localización de los carcinomas del seno maxilar en supra e infraestructura por una línea imaginaria que se extiende desde el canto interno del ojo al ángulo mandibular, la conocida línea de Öhngren ${ }^{10,11}$. Una de las clasificaciones más modernas es la de James Brown del año 2000, donde la clase I implica maxilectomía sin fístula oro-antral, la clase 2 es una maxilectomía baja, que no incluye el piso de la órbita o su contenido, la clase 3 es una maxilectomía alta que involucra el contenido orbitario y la clase 4 incluye además exanteración orbitaria ${ }^{2}$.

En nuestro trabajo utilizamos la clasificación de Cordeiro, que ya detallamos, ya que permite aproximarnos adecuadamente al tipo de reconstrucción necesaria según el tipo de defecto posterior a la maxilectomía ${ }^{5,12}$.

Cordeiro, junto con realizar la clasificación crea un algoritmo reconstructivo, donde dependiendo del tipo de maxilectomía realizada propone ciertas alternativas $^{5,12}$. En los defectos Tipo I, con maxilectomía limitada y resección generalmente de paredes anterior y medial, donde el paladar y piso orbitario están intactos. La reconstrucción de elección es el colgajo radial que brinda una adecuada cobertura externa de piel con un volumen adecuado. En las maxilectomías Tipo II, donde la resección y el defecto son subtotales y la resección puede ser menor 
a $50 \%$ (Tipo IIA) o mayor al 50\% (Tipo IIB) y la recomendación en este caso es con colgajos con dos islas de piel. En el caso del defecto Tipo IIA la reconstrucción puede incluir tanto un colgajo libre o un injerto de piel y una prótesis obturadora, siendo de elección el colgajo libre fasciocutáneo de antebrazo. En defectos Tipo IIB es necesario un colgajo libre osteocutáneo, donde se requiere hueso para proveer soporte estructural y piel para la región del neopaladar y piso nasal. Una prótesis resultaría inadecuada ya que el hueso es necesario para dar soporte al labio superior. El colgajo de elección es el colgajo libre osteocutáneo de antebrazo en sandwich. En la maxilectomía total con defectos Tipo III, donde se involucra la resección de las seis paredes del maxilar; se subdividen en aquellas resecciones que excluyen (Tipo IIIA) o incluyen (Tipo IIIB) el contenido orbitario. En ambos casos se genera un defecto con gran a moderada deficiencia de cobertura y con una gran área a cubrir. En los defectos Tipo IIIA un injerto óseo podría reemplazar el piso orbitario y un colgajo libre con una o más islas de piel podrían reconstruir el paladar, borde nasal y/o mejilla. Los objetivos son lograr soporte del globo ocular, obliterar la comunicación entre la órbita y nasofaringe y reconstruir la superficie del paladar. Injertos de calota, cresta ilíaca o costilla pueden utilizarse para reconstruir prominencia maxilar y piso orbitario. Es de elección el colgajo miocutáneo de recto abdominal, que cubre el injerto óseo y separa los contenidos orbitarios de la cavidad oral. En el caso de pacientes que no son candidatos a colgajos libres es una buena opción el colgajo temporal, que podría cubrir un injerto óseo de haberlo y además da volumen al defecto mediofacial. En defectos Tipo IIIB, con un defecto de cobertura mayor, es de elección el colgajo libre miocutáneo de recto abdominal, con una o más islas cutáneas para reconstruir el paladar, pared nasal lateral y cualquier defecto cutáneo. Por último, en el caso de maxilectomías Tipo IV u orbitomaxilectomías es de elección el colgajo de recto abdominal, con una o más islas cutáneas usadas para cubrir defectos externos de piel o sellar cavidad nasal.

En la experiencia presentada, al clasificar los defectos según la extensión de la maxilectomía realizada podemos ver que en los defectos Tipo IIA o parcial, al ser defectos de coberturas de poca extensión, la prótesis obturadora fue adecuada y permitió separar las cavidades oral y sinonasal sin inconvenientes con un buen resultado funcional. En los pacientes con maxilectomías Tipo IIB fue utilizado el colgajo radial, que es delgado y con un pedículo relativamente largo y permitió el cierre del paladar. Para la reconstrucción post maxilectomía Tipo IIIA, es decir, que no incluye contenido orbita- rio, de los cuatro pacientes en tres pacientes que no eran candidatos a reconstrucción con colgajo libre por su compromiso del estado general y lo avanzado de su enfermedad se reconstruyó con un colgajo pediculado de músculo temporal bilateral logrando cobertura del defecto con un volumen adecuado y en un paciente se utilizó un colgajo libre de latissimus dorsi con buen resultado funcional y estético. Con el colgajo de músculo temporal obtuvimos un resultado adecuado, ya que nos permitió realizar la cobertura del defecto y con baja morbilidad para el paciente, siendo que eran pacientes que no estaban en condiciones de ser sometidos a una cirugía más compleja. En estos casos, nos parece adecuado el uso de este tipo de colgajos en pacientes seleccionados.

En un caso con un defecto que incluyó el contenido orbitario, es decir, Tipo IIIB la reconstrucción fue realizada con un colgajo libre de latissimus dorsi, en el cual se registró una complicación del colgajo, con pérdida parcial de éste debida a congestión venosa, de todas maneras se logró la cobertura adecuada del defecto, con un volumen adecuado, que brindaba tejido muscular y piel.

La reconstrucción de estructuras óseas se logra de manera óptima con colgajos libres vascularizados, tales como fíbula, cresta ilíaca y escápula entre otros. Éstos son útiles en defectos óseos de distintos tamaños y tanto en pacientes que han recibido radioterapia o que la recibirán en el postoperatorio ${ }^{13,14}$. Por otra parte, los injertos óseos no vascularizados son apropiados en defectos estructurales de pequeño tamaño y en pacientes sin radioterapia y con lechos bien irrigados. En nuestra serie de pacientes no utilizamos injertos óseos ni colgajos libres óseos ya que se trataba de pacientes de muy mal pronóstico, con una supervivencia esperada menor al $50 \%$ a los dos años y, por otra parte, se trataba de una experiencia inicial donde se privilegió aplicar la mejor técnica posible con la menor morbilidad para el paciente, es decir, se realizó reconstrucción con los colgajos libres más conocidos por el equipo y que permitieran un resultado adecuado, como es el caso de las reconstrucciones de tercio medio facial donde se prefirió utilizar un colgajo latissimus dorsi por sobre el colgajo de músculo recto abdominal que tal vez estaría más recomendado por la región, pero nos pareció adecuado porque brindaba por una parte seguridad en la toma del colgajo, posee un volumen adecuado para reconstruir el defecto y tiene un pedículo de longitud apropiada para realizar la anastomosis del colgajo.

\section{Referencias}

1. Cordeiro PG, Santamaria E. A classification system and algorithm for reconstruction of maxillectomy and mid- 
facial defects. Plast Reconstr Surg. 2000;105:2331-46.

2. Brown JS. Maxillary reconstruction. Indian J Plast Surg. 2007;40:35-43.

3. Hurvitz KA, Kobayashi M, Evans GR. Current options in head and neck reconstruction. Plast Reconstr Surg. 2006;118:122e-133e.

4. McCarthy CM, Cordeiro PG. Microvascular reconstruction of oncologic defects of the midface. Plast Reconstr Surg. 2010;126:1947-59.

5. Cordeiro PG, Chen CM. A 15-year review of midface reconstruction after total and subtotal maxillectomy: part I. Algorithm and outcomes. Plast Reconstr Surg. 2012;129:124-36.

6. Edge SB, Byrd DR, Compton CC. ed. AJCC Cancer Staging Manual. 7th ed. New York, NY: Editorial: Springer, 2010.

7. Dindo D, Demartines N, Clavien PA. Classification of surgical complications: a new proposal with evaluation in a cohort of 6336 patients and results of a survey. Ann Surg. 2004;240:205-13.

8. Cordeiro, PG, Disa, JJ. Challenges in midface reconstruction. Semin Surg Oncol. 2000;19:218-25.
9. Dalgorf D, Higgins K. Reconstruction of the midface and maxilla. Curr Opin Otolaryngol Head Neck Surg. 2008;16:303-11.

10. Skorek A. History of treatment and classification of maxillary sinus neoplasms. Otolaryngol Pol. 2005;59:771-6.

11. Ohngren G. Malignant Disease of the Upper Jaw: (Section of Laryngology and Section of Otology). Proc R Soc Med. 1936;29:1497-514.

12. Cordeiro PG, Chen CM. A 15-year review of midface reconstruction after total and subtotal maxillectomy: part II. Technical modifications to maximize aesthetic and functional outcomes. Plast Reconstr Surg. 2012;129:139-47.

13. Andrades $\mathrm{P}$, Militsakh $\mathrm{O}$, Hanasono MM, Rieger J, Rosenthal EL. Current strategies in reconstruction of maxillectomy defects. Arch Otolaryngol Head Neck Surg. 2011;137:806-12.

14. Andrades P, Rosenthal EL, Carroll WR, Baranano CF, Peters GE. Zygomatic-maxillary buttress reconstruction of midface defects with the osteocutaneous radial forearm free flap. Head Neck 2008;30:1295-302. 